\title{
Mittheilungen aus dem Laboratorium
} von Prof. Dr. J. W islicenus in Zürich. (Eingelaufen den 21. Juli 1872.)

\section{Ueber die Einwirkung von Natrium auf krystallinisches Dibrombenzol;

$$
\text { von Dr. F. Riese *). }
$$

Im Jahre 1869, in welchem die nachstehend referirten Untersuchungen ausgeführt wurden, war noch einiger Grund vorhanden, das bei der Einwirkung von Brom auf Benzol in der Wärme entstehende Dibrombenzol als die Ortho-Verbindung anzusehen. Seither mufste allerdings diese Meinung fallen gelassen werden, namentlich als V. Me yer $* *$ ) durch Umwandlung desselben in ein Dimethylbenzol und Oxydation des letzteren zu Terephtalsäure die $1: 4$ oder Para-Stellung der beiden Bromatome mindestens höchst wahrscheinlich gemacht hatte. Unter dem Eindrucke der alten, damals noch nicht als unhaltbar erkannten Anschauung schien es mir von Interesse, den Versuch zu machen, durch Einwirkung von Natrium auf das Dibrombenzol ein polymeres Phenylen darzustellen. Möglicherweise konnten drei Gruppen des letzteren

*) Die beiden nachstehenden Arbeiten des Herm Riese, in der Inauguraldissertation des Verfassers um Ostern 1869 publicirt, sind nur durch kurze Referate in den Berichten der deutschen chemischen Gesellschaft und der Zeitschrift für Chemie in weiteren Kreisen bekannt geworden. Zwei Anfragen von Fachgenossen bestimmen mich, noch nachträglich die in Folge Verlustes eines für die Annalen bestimmten Manuscriptes unterlassene ausführlichere Veröffentlichung nachzuholen.

J. $\boldsymbol{W}$.

**) Berichte der deutschen chemischen Gesellschaft $\mathbf{3}, 753$.

Annal. d. Chem. u. Pharm. CLXIV. Bd. 
zu einem Molecule zusammentreten und dadurch einen Kohlenwasserstoff von der Formel $\mathrm{C}_{18} \mathrm{H}_{12}$, das Chrysen oder ein Isomeres liefern :
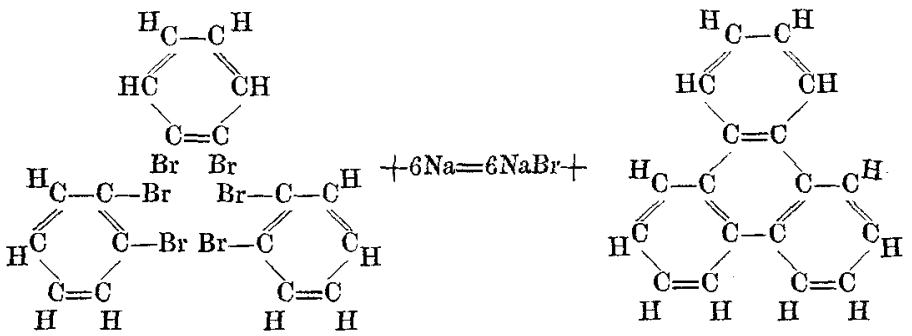

Die angestellten Versuche haben allerdings, wie heute leicht vorauszusehen wäre, zu dem gehofften Resultate nicht geführt, indefs doch einige Ergebnisse geliefert, welche der Veröffentlichung werth sein mögen.

Da ich zunächst gröfsere Mengen von Dibrombenzol darzustellen hatte, bot sich mir die Gelegenheit, neben dem bekannten krystallinischen Körper die Bildung eines isomeren Productes bei der Einwirkung von Brom auf Benzol nachzuweisen.

Das von Riche und Bèrard *) angegebene Verfahren, Kochen von Benzol mit einem Bromüberschusse während längerer Zeit, ist jedem anderen vorzuziehen. Da Caoutchoucund Korkverbindungen nicht anwendbar sind, so ersetzte ich den Rückflufskühler durch eine an den verengten Hals einer grofsen Retorte angeschmolzene ein Meter lange Glasröhre, welche mit Fliefspapier umwickelt und durch auftropfendes Wasser gekühlt wurde. Nachdem die Materialien, 1 Theil Benzol auf 8 Theile Brom, durch ein langes Trichterrohr unter guter Abkühlung eingetragen worden waren, wurde mit aufwärtsgerichtetem Rohre 36 bis 48 Stunden lang im

*) Diese Annalen 138, 51 . 
Sieden erhalten; die entweichenden Bromwasserstoffdämpfe wurden durch Wasser absorbirt. Später wurde der verlängerte Retortenhals abwärts gerichtet, der Bromüberschufs gröfstentheils abdestillirt und das rückständige Flüssigkeitsgemenge ausgegossen. Beim Erkalten schied dasselbe eine reichliche Krystallisation von Dibrombenzol aus, von welchem der flüssig gebliebene Antheil, welcher noch viel Brom enthielt, abgegossen wurde. Durch wiederholtes Abdestilliren und darauf folgendes Schütteln mit heifser Natronlauge wurde das Brom vollständig entfernt und die helle ölige Flüssigkeit der Krystallisation überlassen. Das reichlich ausgeschiedene krystallinische Dibrombenzol war noch von einer gelblichen öligen Flüssigkeit ${ }^{*}$ ) durchdränkt, von welcher es durch scharfes Auspressen befreit werden konnte. In ihr fand sich das isomere Dibrombenzol in noch unreinem Zustande.

Zur Reinigung des krystallinischen $\alpha$-Dibrombenzols wandte ich mit bestem Erfolge die Destillation mit Wasser an, mit dessen Dämpfen es sehr reichlich und von Anfang bis Ende schneeweirs übergeht, während im Destillationsgefässe eine schwarze schmierige Masse zurückbleibt. Einmaliges Umkrystallisiren aus kochendem Weingeist genügt, um es rein und in prachtvollen Krystallen zu erhalten. Es schmilzt nun bei $89^{\circ}$ und siedet bei $219^{\circ}$ (corrig.), wie auch von $\mathrm{C}$ ouper schon angegeben worden ist.

Zur Ausführung der Eutbromung des Dibrombenzols empfahl sich selbstverständlich das treffliche, von Fittig bei seinen Synthesen aromatischer Kohlenwasserstoffe angewendete und später verbesserte ${ }^{* *}$ ) Verfahren.

*) $\beta$-Dibrombenzol. Siehe die folgende Mittheilung.

**) Diese Annalen 144, 277 . 
In Aether, der durch mehrtägiges Stehen über Chlorcalcium und Abdestilliren über demselben entwässert worden war, wurde etwa das doppelte der für das anzuwendende Dibrombenzol theoretisch nothwendigen Menge Natrium in feinen Scheibchen eingetragen. Der Aether wurde am aufgerichteten Kühler einige Zeit mit dem Natrium im Sieden erhalten, weil sich beim Erwärmen noch lebhafte Wasserstoffentwickelung zeigte, wenn auch in der Kälte das vollständige Aufhören derselben die Bindung alles Wassers und Alkohols anzudeuten schien. Es wurde sodann das im Luftbad bei 50 bis $60^{\circ}$ sorgfältig getrocknete Dibrombenzol unter äufserer Abkühlung eingetragen. Unerwarteterweise erwies sich letztere als völlig überflüssig, indem sogar nach mehrstündigem Erhitzen auf dem Wasserbad die Reaction nur sehr allmälig eintrat. Der Beginn derselben charakterisirt sich durch das Erscheinen kleiner schwarzer Punkte auf dem Natrium, die allmälig an Zahl zunehmen, wobei die Flüssigkeit in mäfsiges freiwilliges Sieden geräth, bis schliefslich nach etwa 5 bis 7 Stunden das Metall ganz mit einer aufgelockerten grünlichschwarzen Masse bedeckt ist und mit Beendigung der Reaction der Kolbeninhalt sich wieder abkühlt. Bei dem voraussichtlich hohen Siedepunkt des zu erwartenden Kohlenwasserstoffs erschien es zweckmäfsiger, durch Lösungsmittel denselben vom unangegriffenen Natrium und Bromnatrium zu trennen, statt nach Fit tig das Product unmittelbar abzudestilliren. Der Kolbeninhalt wurde somit mehrmals mit entwässertem *) Aether ausgezogen, der Aether verdunstet und eine Probe des braunen halbfesten Rückstandes mit eingesenktem Thermometer destillirt. Nachdem gegen $100^{\circ}$ einige Tropfen

*) Gewöhnlicher käuflicher Aether erwies sich hierzu als unbrauchbar, da beim Zusammenkommen damit die Masse aufquoll und eine schlammige Beschaffenheit annahm, die die Extraction sehr hinderte. 
einer farblosen Flüssigkeit, die nach Geruch und Flüchtigkeit sich als Benzol erwies, übergegangen waren, zeigte sich erst gegen $300^{\circ}$ eine geringe Menge eines im Retortenhalse krystallinisch erstarrenden, kaum gefärbten Destillats. Nachdem hierauf die Temperatur bis über den Siedepunkt des Quecksilbers gestiegen war, ging wiederum ein wenig eines festen, bei ziemlich hoher Temperatur schon erstarrenden, stark gefärbten und etwas schmierigen Productes über. Die Hauptmasse des Retorteninhaltes erwies sich aber als nicht unzersetzt flüchtig; sie färbte sich schwarz, entwickelte reichlich Bromwasserstoff und hinterliefs schliefslich beim Erhitzen bis fast zum Glühen viel aufgeblähte Kohle.

Die geringe Menge des zuerst übergegangenen krystallinischen Products liefs sich aus Alkohol umkrystallisiren und zeigte sich frei von Brom*), wogegen eine Probe des ursprünglichen Reactionsproductes einen sehr starken Gehalt an demselben ergab.

Da somit nicht alles Brom des Dibrombenzols durch das Natrium herausgenommen worden war, woraus sich auch die geringe Energie der Reaction erklärt, so versuchte ich durch Veränderung der Bedingungen ein günstigeres Resultat zu erzielen.

Ein Versuch, als Lösungsmittel des Dibrombenzols Benzol wegen seines höheren Siedepunktes, der eine energischere Einwirkung erwarten liefs, zu verwenden, zeigte die auffallende Thatsache, dafs selbst nach sechsstündigem Sieden gar keine Einwirkung stattfand, vielleicht in Folge eines geringen gelben Ueberzugs, mit dem das Natrium sich bald bedeckte.

*) In derartigen Fällen prüft man auf Brom am Einfachsten durch Zusammenschmelzen mit einem Körnchen Natrium; nach dem Lösen in Wasser und Ansäuern wird das Brom durch Silberlösung nachgewiesen. 
Bei directem Zusammenschmelzen von Dibrombenzol und Natrium verlief dagegen die Reaction zu energisch, unter $\mathbf{E r}$ glühen und Verkohlung der Masse.

Ein Versuch mit feinpulverigem Silber, in welchem $W$ islicenus*) für die Gruppe der Fettkörper ein treffliches Mittel zur Verkettung der Molecule durch Herausnahme von Jod gefunden hat, ergab nur ein negatives Resultat, indem dasselbe mit Dibrombenzol zum Sieden erhitzt ganz unverändert bleibt, was theils durch die festere Bindung der Halogene in der aromatischen Gruppe, theils durch die geringere Affinität zwischen Silber und Brom als zwischen Silber und Jod sich erklärt. Stärkeres Erhitzen im zugeschmolzenen Rohr wurde nicht versucht, da schon beim Siedepunkt des Dibrombenzols das Silber sich stark zusammenballte und seine für die Einwirkung günstige feine Zertheilung verlor.

Obgleich sich somit kein Mittel finden liefs, das die Bindung alles Broms bewirkt hätte, so schienen mir doch die mittelst Natrium in ätherischer Lösung erhaltenen Producte weiterer Untersuchung werth zu sein. Bei der Unanwendbarkeit der Destillation zu deren Trennung versuchte ich dieselbe mittelst verschiedener Lösungsmittel zu bewerkstelligen. Hierbei zeigte sich bald eine grofse Schwierigkeit, indem die einzelnen Producte in Lösungsmitteln, in denen ein Theil von ihnen isolirt unlöslich ist, sich doch gegenseitig in Lösung zu halten vermögen, während andererseits durch Ausfällen oder Auskrystallisiren eines dieser Körper ein Theil der anderen, der seinen Löslichkeitsverhältnissen nach in dem vorhandenen Mittel gelöst bleiben könnte, mit ausgeschieden wird.

Der dunkelbraune, etwas eingedampfte ätherische Auszug des Reactionsproductes wurde mit viel Alkohol versetzt, wodurch sofort eine gelbbraune amorphe Masse niederfiel, während 
die überstehende Flüssigkeit nur weingelb gefärbt blieb. Aus letzterer wurde durch Eindampen und Erkaltenlassen eine moosartige Krystallisation erhalten, die aber nach dem $\mathbf{A b}$ pressen beim Wiedererhitzen mit Alkohol sich nur theilweise löste, während der Rückstand nur in Aether löslich war. Jede weitere Krystallisation liefs wieder einen solchen Rückstand, so dafs schliefslich fast die ganze Menge in einen in Alkohol leicht- und in einen darin unlöslichen Antheil annähernd getrennt war. Prüfungen auf Brom ergaben in dem ersteren einen ziemlich geringen, im letzteren einen gröfseren Bromgehalt; beide lieferten beim Erhitzen krystallinisch erstarrende Destillate, der schwerer lösliche Theil jedoch unter stärkerer Zersetzung, wonach in denselben die Hauptmenge des in der ursprünglichen alkoholischen Lösung noch vorhandenen amorphen bromhaltigen Productes übergegangen zu sein schien.

Da ich beobachtet hatte, dafs der von den Lösungen abdestillirte Alkohol beim Mischen mit Wasser einige weifse Krystallflitterchen abschied, so glaubte ich erwarten zu können, dafs bei Destillation mit Wasser noch mehr von diesem flüchtigen Körper übergehen werde, wie ja viele Substanzen mit Wasser in weit gröfserer Menge verdampfen als mit Alkohol, so z. B. das Anilin, obgleich dasselbe in letzterem leicht, in Wasser fast unlöslich ist. Der Versuch bestätigte diese Erwartung. Nach Vereinigung der in Alkohol ungelöst gebliebenen Antheile mit dem beim Verdunsten der Mutterlaugen bleibenden Rückstande wurden dieselben in der oben gelegentlich der Reinigung des $\alpha$-Dibrombenzols erwähnten Weise mit Wasser destillirt, so lange noch feste Substanz überging. Es schied sich in der Vorlage ein schneeweifser krystallinischer Körper von aromatischem Geruch $a b$, der abfiltrirt und getrocknet wurde. Derselbe enthielt kein Brom und war unzersetzt flüchtig. Bei der Destillation mit eingesenktem Thermometer, die wegen der geringen Menge Substanz nur annähernde 
Resultate liefern konnte, ging fast die ganze Menge zwischen 237 und $239^{\circ}$ (corr. 242 und $244^{\circ}$ ) über. Eine Bestimmung des Schmelzpunktes ergab denselben bei $69^{\circ}$. Bei der Elementaranalyse erhielt ich folgende Resultate :

0,2599 Grm. Substanz gaben $0,8868 \mathrm{CO}_{2}$, entsprechend $0,2419 \mathrm{C}$, und $0,1540 \quad \mathrm{H}_{2} \mathrm{O}$, entsprechend $0,0171 \mathrm{H}$.

Diese Zahlen, sowie der Schmelzpunkt und Siedepunkt stimmen unerwarteterweise mit der Zusammensetzung und den Eigenschaften des Diphenyls überein :

\begin{tabular}{crrc} 
& \multicolumn{2}{c}{ Berechnet } & Gefunden \\
$\mathrm{C}_{12}$ & 144 & 93,51 & 93,07 \\
$\mathrm{H}_{10}$ & 10 & 6,49 & 6,57 \\
\hline
\end{tabular}

Die Möglichkeiten für die Erklärung der Entstehung desselben aus Dibrombenzol werde ich weiter unten besprechen; jedenfalls ist es nur als ein Nebenproduct der Reaction anzusehen, da seine Menge im Vergleich zum angewandten Material aufserordentlich gering war.

Nach der Entfernung des Diphenyls blieb im Siedegefäfs eine gelbliche amorphe Masse zurück, von der eine Probe beim Erhitzen unter gleichzeitiger Schwärzung ein schon bei hoher Temperatur krystallinisch erstarrendes Destillat gab. Da Versuche, aus der amorphen Masse durch Umkrystallisiren reine Producte zu erhalten, wiederum an den oben erwähnten Schwierigkeiten scheiterten, so mufste der in der Hitze zersetzbare Antheil geopfert werden, um durch Destillation wenigstens die eine Hälfte des Gemisches zu gewinnen. Es wurde daher die ganze Menge desselben destillirt und mittelst eines durch die Retorte geleiteten Kohlensäurestromes das Uebergehen der Dämpfe befördert, da dieselben zufolge ihres offenbar sehr hohen Siedepunktes sich gröfstentheils im Bauch der Retorte schon verdichteten. Es wurde so eine nicht bedeutende Menge einer bräunlich gefärbten, krystallinischen, von einer klebrigen Flüssigkeit durchtränkten Masse erhalten, 
die durch wiederholte Destillation, welche in so kleinem Mafsstabe am besten in einem doppelt gebogenen, zugleich als Siedegefäls und Vorlage dienenden Reagensglase vorgenommen wird, von einem Theil der bromhaltigen Schmieren befreit wurde, indem letztere als zersetzter schwarzbrauner Rückstand hinterblieben. Verschiedene Versuche, den immer noch feuchten und gefärbten krystallinischen Körper völlig zu reinigen, ergaben als zweckmäfsigstes Verfahren, denselben mit etwas Ligroine, worin er schwer-, die schmierige Verunreinigung dagegen leichtlöslich ist, zu einem Brei zu zerreiben und diesen zwischen Fliefspapier auszupressen. Hierauf genügt noch eine Destillation und ein- bis zweimaliges Umkrystallisiren aus Benzol, um den Kohlenwasserstoff rein zu erhalten. Zwei aufeinanderfolgende Krystallisationen zeigten beide den Schmelzpunkt $201^{\circ}$ (corr. 2050). Bei der Verbrennung ergab sich in Folge der Schwerverbrennlichkeit der ausgeschiedenen Kohle bei den ersten Analysen (I. und II.) stets ein zu niedriger Kohlenstoffgehalt.

I. 0,2726 Grm. Substanz gaben $0,9175 \mathrm{CO}_{2}=0,2502 \mathrm{C}$, und 0,1476 $\mathrm{H}_{2} \mathrm{O}=0,0164 \mathrm{H}$.

II. $0,2795 \mathrm{Grm}$. Substanz gaben $0,9517 \mathrm{CO}_{2}=0,2586 \mathrm{C}$, und 0,1547 $\mathrm{H}_{2} \mathrm{O}=0,0191 \mathrm{H}$.

\begin{tabular}{lcc} 
& I. & II. \\
C & 91,79 & 92,88 \\
H & 6,02 & 6,16 \\
\hline & 97,81 & $99,04$.
\end{tabular}

Diese beiden Analysen geben bei ziemlichen Abweichungen im Kohlenstoffgehalt doch sowohl unter sich als mit der nachfolgenden dritten übereinstimmende Zahlen für den Wasserstoff. Es bedurfte bei letzterer der ganzen Hitze des trefflichen Erlenmeyer'schen Ofens, um schliefslich eine vollständige Verbrerınung zu erzielen.

III. $0,1540 \mathrm{Grm}$. Substanz gaben $0,5300 \mathrm{CO}_{2}=0,1445 \mathrm{C}$, und 0,0866 $\mathrm{H}_{2} \mathrm{O}=0,0096 \mathrm{H}_{;}$diels ergiebt den Procentgehalt zu 


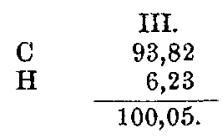

Die hieraus sich berechnende einfachste Formel $\mathrm{C}_{9} \mathrm{H}_{7}$ verlangt 93,91 pC. Kohlenstoff und 6,09 pC. Wasserstoff, während dem Triphenylen, das ich zuerst vor mir zu haben glaubte, 94,74 pC. $\mathrm{C}$ und 5,26 pC. $\mathrm{H}$ zukommen. Die Formel $\mathrm{C}_{9} \mathrm{H}_{7}$ ist übrigens mit Rücksicht auf die physikalischen Eigenschaften dieses Kohlenwasserstoffs, mehr noch aber einem weiter unten zu beschreibenden Versuch zufolge, wonach derselbe als Diphenylbenzol oder Diphenylphenylen $\mathrm{C}_{6} \mathrm{H}_{4}\left\{\begin{array}{l}\mathrm{C}_{6} \mathrm{H}_{5} \\ \mathrm{C}_{6} \mathrm{H}_{5}\end{array}\right.$ aufzufassen ist, auf $\mathrm{C}_{18} \mathrm{H}_{14} \mathrm{zu}$ verdoppeln.

Das Diphenylbenzol schmilzt, wie erwähnt, bei $205^{\circ}$; eine genaue Bestimmung des Siedepunktes war nicht möglich, indem derselbe hoch über $360^{\circ}$ liegt; da es jedoch andererseits in einem Probirröhrchen in den Dampf von siedendem Schwefel $\left(440^{\circ}\right)$ gebracht lebhaft kocht, so lälst sich als Siedepunkt des Diphenylbenzols annähernd $400^{\circ}$ bezeichnen. Es ist selbst in siedendem Alkohol gar nicht, kaum in Aether löslich; etwas mehr wird es von Ligroïne und noch mehr von Benzol aufgenommen, in welch letzterem es in der Hitze leicht löslich ist, so dafs eine kochend gesättigte Lösung beim Abkühlen zu einem compacten Kuchen erstarrt. In hohem Grade wird durch die Anfangs beigemengte Schmiere die Löslichkeit in allen Mitteln erhöht, weshalb auch die Ausbeute an reinem Product aus der ohnehin schon kleinen Menge des ersten rohen Destillats eine sehr geringe ist. Aus verdünnter Lösung in Benzol krystallisirt das reine Diphenylbenzol in kleinen weifsen, büschelförmig gruppirten platten Nadeln. Es besitzt in der Kälte gar keinen und auch erhitzt nur einen schwachen Geruch, ist mit Wasserdämpfen nicht flüchtig und sublimirt schon beim Schmelzpunkt in kleinen irisirenden Flitterchen. 
Das Hauptproduct der Reaction zwischen Natrium und $\alpha$-Dibrombenzol ist übrigens weder das Diphenylbenzol noch das Diphenyl; es sind diefs die bräunlichen, amorphen und bromhaltigen Producte, aus denen es mir nicht gelungen ist, ein einzelnes wohlcharakterisirtes abzuscheiden. Ein grofser Theil derselben bleibt bei der Extraction des Reactionsproductes mit Aether ungelöst zurück, während ein Theil in Lösung geht und durch Zusatz von Alkohol, wie schon erwähnt, gefällt wird. Dabei reifst es eine kleine Menge Diphenyl und Diphenylbenzol mit nieder, die sich durch successive Destillation der Masse mit Wasser und für sich allein erkennen lassen. Der aus der ätherischen Lösung durch Alkohol fällbare und der beim Bromnatrium und Natrium zurückbleibende Körper scheinen ihren Eigenschaften nach dasselbe Product zu sein. Beide sind in Aether und Ligroïne kaum, leicht aber in Benzol und Schwefelkohlenstoff löslich und werden aus diesen Lösungen durch die zuerst genannten Flüssigkeiten wieder unkrystallinisch niedergeschlagen, wobei jedoch eine geringe Menge eines ähnlichen, aber weicheren Körpers in Lösung bleibt; letzterer scheint derselbe zu sein, der in der früher erwähnten alkoholischen Lösung neben Diphenyl und Diphenylbenzol enthalten war. Das mittelst Benzol aus dem Gemisch von Natrium und Bromnatrium ausgezogene und durch Ligroïne wieder gefällte Product, das ein ockerfarbiges, bei $200^{\circ}$ noch nicht schmelzendes, beim Reiben stark electrisch werdendes Pulver darstellt, wurde analysirt.

I. $0,3341 \mathrm{Grm}$. ergaben $0,9493 \mathrm{CO}_{2}=0,2589 \mathrm{C}$, und $0,1224 \mathrm{H}_{2} \mathrm{O}$ $=0,0136 \mathrm{H}$.

II. 0,4189 Grm. nach Carius im zageschmolzenen Rohr oxydirt gaben $0,1750 \mathrm{AgBr}=0,0745 \mathrm{Br}$.

III. 0,4066 Grm. durch Schmelzen mit Soda und Salpeter oxydirt gaben $0,1680 \mathrm{AgBr}=0,0715 \mathrm{Br}$.

Hieraus berechnet sich folgende procentische Zusammensetzung : 


$\begin{array}{lccc} & \text { I. } & \text { II. } & \text { III. } \\ \mathrm{C} & 77,49 & - & - \\ \mathrm{H} & 4,07 & - & - \\ \mathrm{Br} & - & 17,78 & 17,58 .\end{array}$

Auf die Resultate dieser Analysen werde ich unten zurückkommen.

Was nun die Constitution des bereits als Diphenylbenzol bezeichneten Kohlenwasserstoffs betrifft, so war leicht die aus der Analyse gezogene Vermuthung über seine Constitution durch einen Versuch zu prüfen. War die Formel $\mathrm{C}_{6} \mathrm{H}_{4}\left\{\begin{array}{l}\mathrm{C}_{6} \mathrm{H}_{5} \\ \mathrm{C}_{6} \mathrm{H}_{5}\end{array}\right.$ richtig, so war zu erwarten, dafs bei Einwirkung von Natrium auf ein Gemenge von einem Molecul Dibrombenzol mit zwei Moleculen Monobrombenzol eine gröfsere Menge desselben entstehe, als aus ersterem allein, nach der Gleichung :

$$
\mathrm{C}_{6} \mathrm{H}_{4} \mathrm{Br}_{2}+2 \mathrm{C}_{6} \mathrm{H}_{6} \mathrm{Br}+2 \mathrm{Na}_{2}=\mathrm{C}_{6} \mathrm{H}_{4}\left\{\mathrm{C}_{6} \mathrm{H}_{5} \mathrm{H}_{5}+4 \mathrm{NaBr}\right.
$$

Der Versuch bestätigte diese Erwartung vollkommen. Um unter ganz gleichen Bedingungen zu arbeiten, wurde die Reaction auch mit Dibrombenzol alleiu noch einmal wiederholt, damit der Kohlenwasserstoff in beiden Fällen auf genau die gleiche Weise abgeschieden werden konnte. Es wurden einerseits $70 \mathrm{Grm}$. Dibrombenzol, andererseits eben so viel desselben gemischt mit $100 \mathrm{Grm}$. Monobrombenzol in der früher beschriebenen Weise der Einwirkung von Natrium unterworfen. Der Procefs verlief bei Gegenwart von Monobrombenzol viel energischer, so dafs sogar zeitweise äufsere Abkühlung nöthig wurde. Die Producte beider Reactionen wurden dann mit Benzol extrahirt und die nach dem Abdunsten desselben bleibenden Rückstände mit eingesenktem Thermometer destillirt und das bis $300^{\circ}$ Uebergehende, das nur aus Diphenyl bestand, für sich aufgefangen. Die Menge desselben aus den gemischten Bromüren war ziemlich beträchtlich, während aus dem Dibrombenzol allein wieder nur sehr wenig erhalten 
wurde. Dann wurde das Thermometer entfernt, durch den Tubulus ein Strom Kohlensäure durch die Retorte geleitet und weiter erhitzt, bis die flüssige schwarze Masse in der Retorte anfing wieder fester zu werden und in aufgeblähte Kohle überzugehen. Aus dem Gemenge von Mono- und Dibrombenzol wurden so $12,5 \mathrm{Grm}$, aus dem letzteren allein 2,5 Grm. des rohen Kohlenwasserstoffs erhalten. Nach der auf die oben beschriebene Art ausgeführten Reinigung zeigte sich dieser Unterschied weniger stark, jedoch immerhin noch evident, indem in einem Falle 2,4, im anderen 0,9 Grm. des fast absolut reinen Productes erhalten wurden; letzterer Umstand hatte seine Ursache darin, dafs das aus den gemischten Bromüren erhaltene Destillat ein wenig mehr schmierige Beimengung enthielt, die bei der Reinigung einen gröfseren Verlust an krystallinischer Substanz herbeiführte. Dafs die aus beiden Reactionen hervorgehenden Producte identisch sind, bewies eine nach vollständiger Reinigung vorgenommene Schmelzpunktsbestimmung, welche bei beiden $205^{\circ}$ (corrigirt) ergab. Dieses Resultat spricht entscheidend für die aufgestellte Formel. Auch würde vielleicht bei einer geringen Veränderung der Ausführung eine noch weit gröfsere Ausbeute erzielt worden sein; da nämlich die Einwirkung des Natriums auf Dibrombenzol erst nach längerer Zeit, auf Monobrombenzol dagegen sehr bald eintritt, so verging jedenfalls einige Zeit, in der nur das letztere derselben ausgesetzt war, woher auch die bedeutende Menge Diphenyl stammt; erst später, als nur noch wenig Monobrombenzol vorhanden war, begann die Betheiligung des Dibrombenzols an der Reaction nnd damit auch die Bildung des Diphenylbenzols. Diefs wäre vielleicht dadurch zu vermeiden, dafs das erstere erst dann allmälig zugesetzt würde, wenn die Einwirkung auf das Dibrombenzol bereits begonnen hätte. 
Neben diesem synthetischen Versuch hätte vielleicht auch ein anderer mehr analytischer eine weitere Bestätigung der aufgestellten Formel geliefert; ein 0 ydationsversuch hätte nämlich vielleicht durch Bildung einer Phtalsäure, wahrscheinlich Terephtalsäure, das Vorhandensein zweier Seitenketten noch evidenter dargethan. Leider reichte jedoch die geringe Menge Material hierzu nicht aus. Dagegen ergeben sich auch noch weitere Gründe für die Formel $\mathrm{C}_{6} \mathrm{H}_{4}\left\{\begin{array}{l}\mathrm{C}_{6} \mathrm{H}_{5} \\ \mathrm{C}_{6} \mathrm{H}_{5}\end{array}\right.$. Das Diphenyl, das mit dem Diphenylbenzol insofern grofse Aehnlichkeit in der Constitution hat, als es einfach-, letzteres zweifach-phenylirtes Benzol darstellt, bildet mit Pikrinsäure keine Verbindung; eben so gelang es mir auch nicht, durch Vermischen einer gesättigten Lösung meines Kohlenwasserstoffs in Benzol mit einer concentrirten Pikrinsäurelösung eine krystallinische Abscheidung zu erhalten. Endlich erlauben auch die Siedepunktsverhältnisse vielleicht einen Schlufs in dieser Richtung. Benzol siedet bei $82^{\circ}$, Diphenyl bei $240^{\circ}$ und Diphenylbenzol bei etwa $400^{\circ}$; die Differenzen in dieser Reihe betragen je etwa $160^{\circ}$, um welche Zahl sonach der Eintritt je eines Phenyls in das Benzol den Siedepunkt zu erhöhen scheint.

Die Frage nun, in welcher Weise die beiden Kohlenwasserstoffe im vorliegenden Falle entstanden seien, ist mit völliger Sicherheit jedenfalls nicht zu entscheiden, sondern läfst nur Vermuthungen zu. Das Eigenthümliche und Unerwartete, die Bildung des Phenylrestes statt des a priori allein zu erwartenden Phenylens, läfst sich jedenfalls nicht durch die einfache Annahme eines Gehaltes des Dibrombenzols an Monobrombenzol erklären, da letzteres nach dem mitgetheilten Reinigungsverfahren vollständig ausgeschlossen war; eben so wenig genügt die Annahme einer Bildung von Monobrombenzol aus dem Dibrombenzol durch Wasserstoff im Status 
nascens, der aus nicht völlig entwässertem Aether entstanden sein könnte. Die Bildung der beiden Kohlenwasserstoffe mufs somit jedenfalls mit der der brombaltigen Producte in Zusammenhang gebracht werden. Es geschah nur in dieser Absicht, dafs ich die angeführte Analyse derselben ausgeführt habe, die ja bei dem völligen Mangel an Kennzeichen einer reinen Substanz sonst keinen Werth haben konnte. Die bei der Analyse gefundenen Zahlen ( $\mathrm{C}=7 \mathbf{7 7 , 5} \mathrm{pC}$., $\mathrm{H}=4,1 \mathrm{pC}$. und $\mathrm{Br}=17,7 \mathrm{pC}$.) stimmen annähernd zur Formel eines einfach-gebromten polymeren Phenylens, des Monobrompentaphenylens, $\mathrm{C}_{30} \mathrm{H}_{19} \mathrm{Br}$, oder $\left(\mathrm{C}_{6} \mathrm{H}_{4}\right)_{4}\left(\mathrm{C}_{6} \mathrm{H}_{3} \mathrm{Br}\right)$, welches 78,43 pC. $\mathrm{C}, 4,14 \mathrm{pC}$. $\mathrm{H}$ und $17,43 \mathrm{pC} . \mathrm{Br}$ verlangt. Angenommen, es läge wirklich dieser Körper vor, so liefse sich die Reaction so erklären, dafs zunächst aus 5 Moleculen Dibrombenzol Pentaphenylen entstanden wäre, welches dann mit einem weiteren Molecul Dibrombenzol zu Monobrompentaphenylen und Monobrombenzol sich umgesetzt hätte. Das letztere hätte dann die Veranlassung zur Bildung der beiden Kohlenwasserstoffe gegeben, wie folgende Gleichung ausdrückt : ${ }_{25} \mathrm{C}_{6} \mathrm{H}_{4} \mathrm{Br}_{2}+23 \mathrm{Na}_{2}={ }_{4} \mathrm{C}_{90} \mathrm{H}_{19} \mathrm{Br}+\mathrm{C}_{6} \mathrm{H}_{4}\left\{\mathrm{C}_{6} \mathrm{C}_{5} \mathrm{H}_{5}+{ }_{\mathrm{C}_{6}}^{\mathrm{C}_{6} \mathrm{H}_{5}}+46 \mathrm{NaBr}\right.$.

Wenn auch diese etwas monströse Gleichung nicht beanspruchen kann, den wirklichen Gang der Reaction auszudrücken, so kann sie doch ein ungefähres Bild derselben geben; sie thut diefs auch einigermafsen in Bezug auf die Mengenverhältnisse der Producte, die hiernach sich verhalten wie 240 Theile Diphenylbenzol und 154 Theile Diphenyl zu 1519 Theilen des bromhaltigen Productes, ein Verhältnifs, das mit dem thatsächlich vorhandenen nach ungefährer Schätzung ziemlich im Einklang stehen mag.

Schliefslich sei noch erwähnt, dafs es mir nicht gelungen ist, durch Erhitzen der bromhaltigen Producte mit Natrium und Aether oder Benzol als Lösungsmittel auf $150^{\circ}$ eine Ent- 
bromung derselben zu bewerkstelligen, was nicht auffallen kann, nachdem Fittig *) gefunden, dafs aus Dibromdiphenyl das Brom sich durch Natrium nicht herausnehmen läfst, und nachdem $W$ ahlforfs $* *$ ) beim Monobromnaphtalin Einwirkung des Natriums erst in der Hitze beobachtet hat. Es ergiebt sich hieraus, dafs die Festigkeit der Bindung der substituirenden Halogenatome bei den aromatischen Kohlenwasserstoffen mit deren Moleculargröfse wächst.

\section{Ueber Betadibrombenzol;}

von Demselben.

Das in vorstehender Abhandlung erwähnte, bei der Darstellung von krystallinischem Dibrombenzol als Nebenproduct erhaltene Oel, welches etwa ein Fünzehntel vom Gewichte des Hauptproductes ausmachte, hielt ich Anfangs für Nonobrombenzol, überzeugte mich aber sofort von der Unrichtigkeit dieser Vermuthung, als ich dasselbe der Destillation unterwarf. Bei $154^{\circ}$, dem Siedepunkte des Monobrombenzols, kochte die Flüssigkeit noch gar nicht; erst bei etwas über $200^{\circ}$ gingen die ersten Tropfen über. Das Thermometer stieg nun langsam bis über $240^{\circ}$, wo unter starker Entwickelung von Bromwasserstoff und Verkohlung des Rückstandes Zersetzung eintrat. Das gelbliche Destillat enthielt noch festes Dibrombenzol, welches bei der gerade herrschenden Winterkälte von $-6^{0}$ sich reichlich ausschied. Die abgegossene

*) Diese Annalen $1 \mathbf{3 2} 205$.

**) Zeitschrift für Chemie 1865, 3 . 